\title{
The Electromagnetic Spectrum: Knowledge and Experimental Techniques
}

\author{
Prof. Samir A. Hamouda ${ }^{* 1}$, Maqboula Khamis Ibrahim ${ }^{2}$, Mohamed Belhasan Mohamed ${ }^{3}$ \\ ${ }^{1}$ Professor, ${ }^{2}$ M.Sc.Scholar, ${ }^{3}$ Ph.D.Scholar \\ Physics Department, University of Benghazi, Libya \\ Corresponding Author Email id: dr_s_hamouda@yahoo.ie
}

\begin{abstract}
The electromagnetic spectrum classifies and explores the properties of electromagnetic radiation. This classification does not contradict the concept of wave and particle, only the type of interaction between radiation and the medium can produce accurate interpretation of the final experimental results. This paper outlines the most relevant experimental techniques evolved from understanding the electromagnetic spectrum and their technologies.
\end{abstract}

Keywords - Methods and Technologies; Vibration; Rotation; Visible Light; Spectroscopy.

\section{Introduction}

The electromagnetic spectrum summarizes all aspects and properties of electromagnetic waves or photons through space. This spectrum allows for exploring relevant experimental methods and new technologies. The electromagnetic spectrum is the entire continuous range of electromagnetic radiation extending from radio waves to gamma rays according to wavelength, frequency and photon energies. It is frequently referred to in the study of science and researches. Therefore, it was thought that this topic would be of great interest to researchers to have basic ideas about this topic and provides reasonable amount of information for useful scientific discussion. This paper presents a brief overview of the general aspects of the electromagnetic spectrum and its uses in various desplines of science.

\section{The Electromagnetic Spectrum}

An electromagnetic wave consists of electric field components and magnetic field components perpendicular to each other and moving with speed of light [1]. The electromagnetic spectrum comprises the spectrum of energy ranging from very long radio waves to very short gamma rays. The human eye can detect and sense only a small portion of this spectrum called visible light. Figure1 shows the bands of the electromagnetic spectrum by common names. It can be stated that there is no sharp cut between bands but the differences between bands are rather gradual.

This study presents the electromagnetic spectrum and summarizes in brief all aspects and properties of electromagnetic waves or photons through space. However, emphasis will be placed on the optical properties of visible light. Light is the small part of the electromagnetic spectrum of various wavelengths. The range of wavelengths starts the infrared portion to the ultraviolet portion.

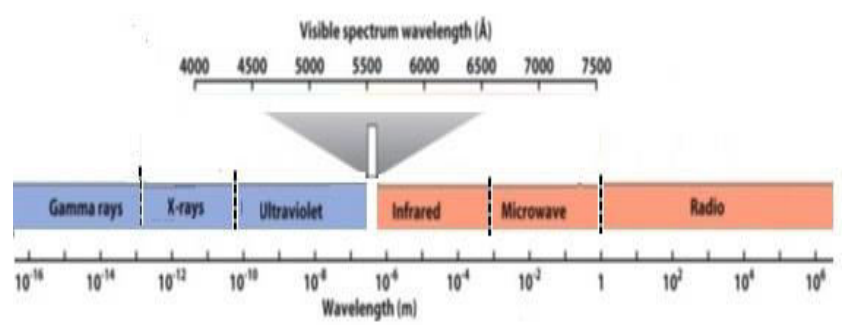

Fig.1: Bands of the electromagnetic spectrum by common names

\section{Experimental Techniques}

In physics, high gamma ray energies are used in Compton (inelastic) scattering techniques for determining the dynamics of electrons in in metals and alloys. When unpolarized photons are Compton scattered, the analysis of the observed energy spectrum provides information about the dynamic distribution of electrons in condensed matter [2]. On the other hand, high intensity of circular polarized photons (Synchrotrons Radiation) has been used to study large classes of magnetic materials [3].

In the earth and space science, electromagnetic radiation is often a part of a deep knowledge about scientific instruments that use the full range of the electromagnetic spectrum to study rocks and minerals, earth's atmosphere, the solar system, cosmic rays, earth's magnetic field, and the universe, and analyzing incoming light (redshift and blue shift) from stars by optical and radio telescopes $[4,5]$.

In biology, it is often involves the interaction of electromagnetic radiation with living organisms and

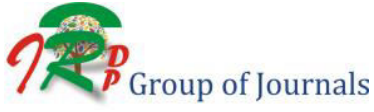


mutagenic sources investigations, the physiology of the eye investigation, and the study of photosynthesis process in plants and trees. In medicine it is often a part of medical diagnosis, treating cancer and curing tumors. X-Rays and MRI are used to get an image of broken bones, injured tissues and locating body disorder. On the other hand, due to their high penetrating energy, gamma rays are used in the medical industry in the treatment of cancerous cells and curing the diseases [4-6].

In chemistry, the electromagnetic spectrum is often discussed when taking about the Vibrational spectroscopy: This method is concerned with the study of absorption of infrared radiation, which causes vibrational transition in the molecules and compounds. Infrared rays are naturally heat energy. This thermal energy induces molecular vibrations in chemical bonds, which can be seen as springs connecting two masses or two atoms (see figure2). Each bond responds to (absorbs) certain resonance frequencies. However, most useful bands in an infrared spectrum correspond to stretching frequencies [7-9].

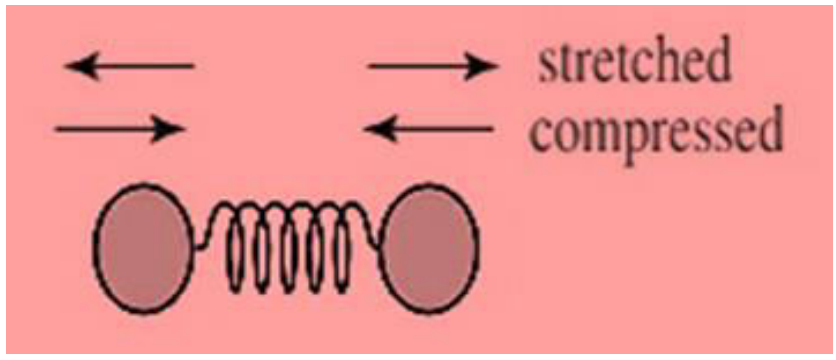

Fig.2: In vibrational spectroscopy, chemical bonds are represented by springs

Rotational spectroscopy: This technique is used for energy analysis of rotational states of molecules and atoms in the gas state.. The method assumes that a molecule must have a transitional dipole moment that is in resonance with the applied electromagnetic field for rotational spectroscopy to be used (see figure3). The spectrum of rotational states energies of polar molecules can be measured in absorption or emission by microwave radiation [7, 10-12].

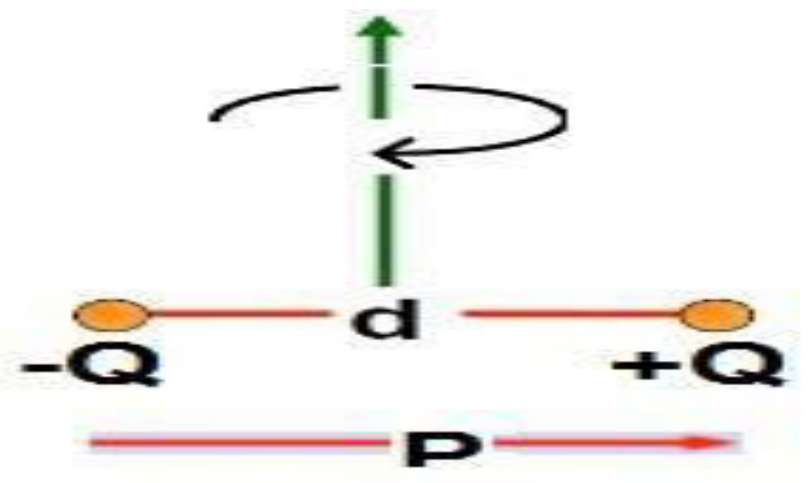

Fig.3: The electric dipole $P=q d$
Electronic spectroscopy: is an analytical technique to study the interaction of molecules with UV lights. Absorption of UV photon results in electronic transition of atoms and molecules, and electrons are excited from ground state to higher electronic states. The energy of UV light can eject an electron from an inner-shell orbital of an atom (see figure4) [13.14].

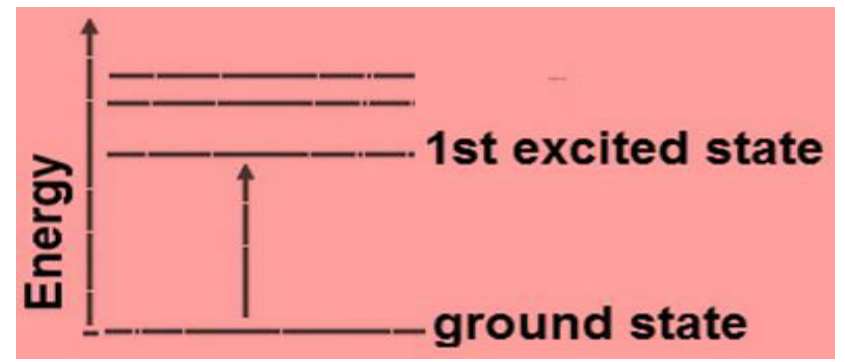

Fig.4: Energy levels of a molecule. Electrons are excited from ground to higher electronic states

$X$-ray photoelectron spectroscopy $(X P S)$ : is a surface analysis technique that can be used to analyse the elemental composition of the surface of materials after treatment. It is an ultra-high vacuum technique and probes surfaces to a depth of $10 \mathrm{~nm}$ of the material. The basic principle of XPS is the photoelectric effect (see figure5).

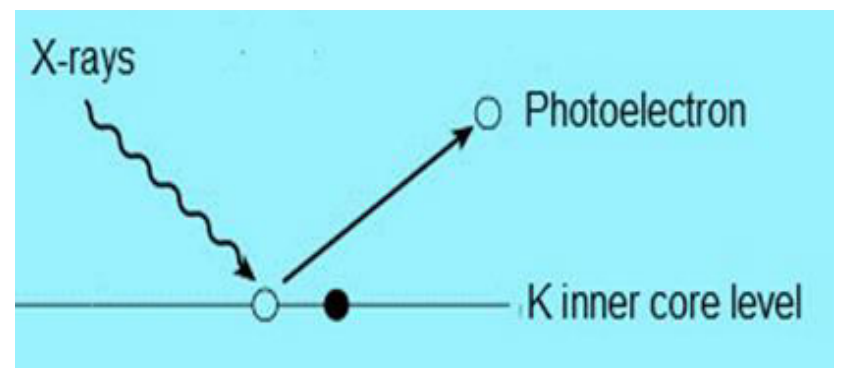

Fig.5: Schematic diagram of photoelectrons process used for XPS

Photoelectrons are emitted from a sample surface as a result of interaction of $x$-ray of characteristic energy line $($ Alk $\alpha 1,1.5 \mathrm{keV})$ with the electrons within the surface of a sample. The kinetic energy of photoelectrons emitted from the surface provides information on the electron binding energy states of atoms in the surface $[15,16]$.

Gamma-ray spectroscopy: is a technique used for the analysis of the energy spectra of gamma-ray sources. The gamma-ray spectrum is characteristic of the gammaemitting nuclides of various energies and intensities contained in the radioactive sources. Therefore, gamma-ray spectroscopy is a vital tool in radiometric examination. Analysis of gamma-ray spectrum is used to determine the elemental and quantity of gamma emitters present in a gamma source (see figure6). However, quality of gamma 
spectrum depends on the quality and performance of gamma-ray detection systems (resolution, signal/background ratio and efficiency) [17-20].

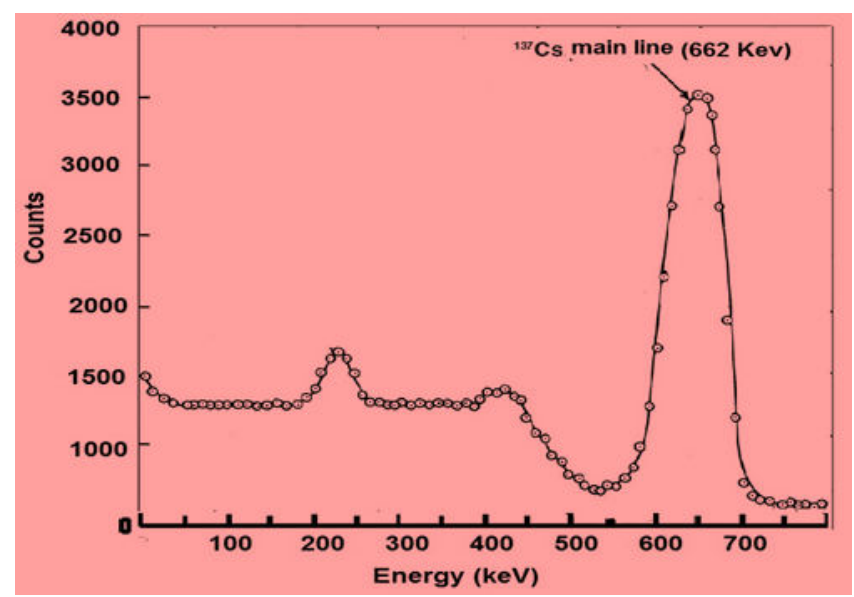

Fig.6: A typical Nal(TI) spectrum for 137Cs source

\section{Properties of Light}

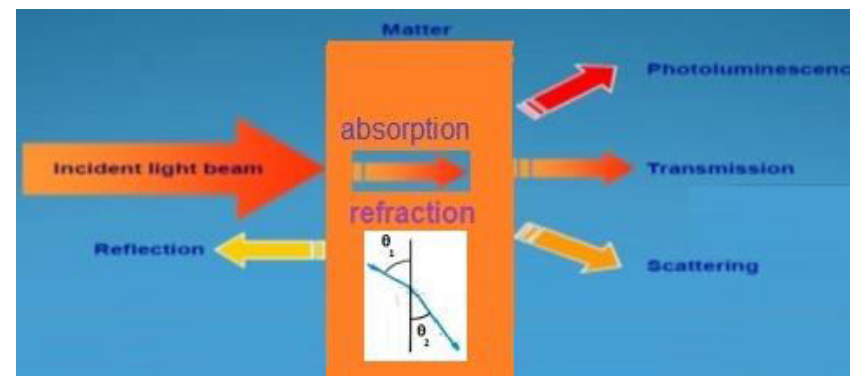

Fig.7: The mechanisms of light interaction with matter

Figure 7 summarizes the mechanisms of light interaction with substances. Refraction: is the change in direction of the incident light at an angle to a boundary (interface) between two transparent media with different densities. Reflection: when light is incident on an interface between media that differ in refractive index, all of the light will be reflected back into the medium from where it travels. The Reflected light comes out at same angle as incident beam, but on other side of normal. Diffraction: Is the bending of a parallel beam of light as it passes through a narrow opening (slit). However, the intensity of the diffraction is related to the slit opening. High intensity is maintained when the slit opening is amounting to the wavelength of light applied. Such a condition allows for constructive interference of light waves resulting in high diffraction intensity. Otherwise, no diffraction will take place for other slit widths. Absorption: When a light beam is incident on a material surface, part of the incident beam is absorbed through the material. Transmission: When a light beam is impinged on a material surface, fraction portion of the incident beam is transmitted through the material. Scattering: Rayleigh scattering and Compton scattering (inelastic scattering). Rayleigh scattering: where a photon interacts with the electrons orbiting an atom and is scattered without any change in photon energy (elastic scattering). Compton scattering: where a photon transfers some of its energy to the atomic electron. The transferred energy should exceed the electron binding energy [21]. Photoluminescence: is the emission of light which is caused by the irradiation of a substance with other light. Figure3: summarizes the mechanisms of light interaction with substances.

\subsection{Ultraviolet Light}

Ultraviolet light is part of the electromagnetic spectrum and lies between the visible portion of the spectrum and the $\mathrm{x}$-ray portion. In physics, the wavelengths of ultraviolet radiation are divided into four regions: near (400-300 nm), middle (300-200 nm), far (200-100 nm), and extreme (below $100 \mathrm{~nm}$ ) [22]. In biology, wavelengths of ultraviolet radiation are divided into three regions designated as: UVA (400-315 nm), which almost 99 percent does reach Earth's surface; UVB (315-280 nm), which can cause strong sickness effects on organisms; and UVC (280-100 nm), which is absorbed in air and has little effects on biological systems [22, 23]. Ultraviolet and Visible light are important tools for the characterization of optical constants (reflectivity, absorption, and transmission, extinction coefficient, index of refraction) of materials. Absorption technique is based on measuring the amount of light absorbed by a liquid, a solid, or a gas sample at a given wavelength [24].

\subsection{Infrared light}

Infrared light is part of the electromagnetic spectrum and lies just to the left of the red end of the visible light spectrum. The wavelengths of Infrared waves range from $(1000-0.7 \mu \mathrm{m})$. The Sun emits all kinds of electromagnetic energy. The visible light reach Earth's surface from the sun amounts to about $(42.3 \%)$. ultraviolet light $(8 \%)$, and infrared light (49.4\%) [25]. Infrared radiation spectroscopy (IR spectroscopy) is widely used in physics, astronomy, biology, medicine, chemistry, industry and forensic science. It is used in study and analysis of complex mixtures of molecular composition. This technique is based on recording of absorption and emission spectra in the Infrared radiation range [26].

\section{Conclusion}

The present study focuses on reviewing the optical techniques that evolved from the knowledge that gained through understanding the properties of the electromagnetic waves. The quality and benefits of techniques are linked to several spectroscopy methods, such as: non-destructive 
measurement, non-invasive measurement, rapid evaluation, and cost-effective accomplishment. Advancements in these analytical methods were due to the fact that significant progress has been made in digital imaging and in a variety of modern optical spectrometer assemblies. On the other hand, the optical spectroscopy is used for obtaining information about a structure and properties of a wide range of materials. It is used to determine some important parameters such as the refractive index, absorption coefficient, extension coefficient, the energy gap, and the dielectric constant (real and imaginary). It is also used for nanomaterial, pharmaceutical, and forensic investigations. Therefore, it is strongly recommended that researchers should acquire knowledge to various types of optical techniques and be able to distinguish and interpret continuous and discrete spectra.

\section{References}

[1] Electromagnetic spectrum - https://www.google.com/ search?q= electromagnetic+spectrum\&oq=Electromagnetic + spectrum\&aqs $=$ chrome.0.0i433i512j0i51219.1771j0j7\&sourceid $=\mathrm{ch}$ rome\&ie=UTF-8, accessed on 10.09.2021

[2] Samir. A. Hamouda, (2016), "Gamma-Ray Compton Spectroscopy of Tungsten Using 662 Kev Gamma-Ray Radiation”, Mordovia University, Bulletin, Vol. 26, no. 2, 211-217

[3] Samir A Hamouda, et al. (2018)," Circular Polarization Of Photons For Magnetic Materials Studies", International Journal Of Core Engineering \& Management, Volume-4, Issue-12.

[4] MSc Chemistry Paper-IX Unit-2.pdf (nou.ac.in), accessed on 11.09.2021.

[5] Microsoft PowerPoint - MODULE 25 (nptel.ac.in), accessed on 11.09.2021.
[6] Kevin V. Hackshaw., et al., (2020)," Vibrational Spectroscopy for Identification of Metabolites in Biologic Samples"., Molecules, 25, 4725; doi:10.3390/molecules25204725 molecules-25-04725 (1).pdf.

[7] Electrophoresis (tanta.edu.eg), accessed on 10.09.2021.

[8] Rotational_1-ARE.ppt (dcu.ie), accessed on 10.09.2021.

[9] Untitled Document (nptel.ac.in), accessed on 12.09.2021.

[10] MSc Chemistry Paper-IX Unit-4.pdf (nou.ac.in), accessed on 11.09.2021.

[11] Slide 1 (uic.edu), accessed on 13.09.2021.

[12] PowerPoint Presentation (nottingham.ac.uk) accessed on 12.09.2021.

[13] X-Ray Photoelectron Spectroscopy - an overview | ScienceDirect Topics accessed on 10.09.2021.

[14] GammaExp-min.pdf (physlab.org), accessed on 14.09.2021.

[15] Physics of Gamma-ray Spectroscopy Measurements (ictp.it), accessed on 10.09.2021.

[16] 0751 - H122 - Basic Health Physics - 22 - Gamma Spectroscopy Overview. (nrc.gov), accessed on 10.09.2021

[17] $137 \mathrm{cs}$ spectrum with mca - https://www.gammaspectacular. com/blue/cs137-spectrum, accessed on 14.09.2021.

[18] Light-Poster.pdf, accessed on 11.09.2021.

[19] 24 Electromagnetic waves.pdf (wright.edu), accessed on 11.09.2021.

[20] Electromagnetic spectrum - https://www.google.com/search? $\mathrm{q}=$ electromagnetic + spectrum \&oq=electromagnetic + spectrum\&aqs $=\mathrm{c}$ hrome. $0.69 \mathrm{i} 59 \mathrm{j} 0 \mathrm{i} 51219.1400 \mathrm{j} 0 \mathrm{j} 4$ \& sourceid = chrome\&ie=UTF-8, accessed on 14.09.2021.

[21] Optical properties (nptel.ac.in), accessed on 11.09.2021.

[22] Ultraviolet radiation | Definition, Examples, Effects, Wavelengths, Types, \& Facts | Britannica

[23] B L Diffey., (1980), "Ultraviolet radiation physics and the skin", Review Article., Phys. Med . Biol., V01 . 25 . No . 3. 405-426. Printed in Great Britain

[24] UK_ANDO_Solis-S_3AN.pdf (rackcdn.com)

[25] JPSS Understanding Infrared Light_Teacher-Parent Activity Manual_Final.pdf (noaa.gov), accessed on 12.09.2021.

[26] Infrared Radiation (issp.ac.ru), accessed on 14.09.2021. 